\title{
Evaluation of an Electronic Smart-Card Based School Absenteeism Surveillance System
}

\author{
Dennis Ip, Eric H.Y. Lau*, Yat-hung Tam, Teresa So, Chi-kin Lam, Benjamin J. Cowling \\ and Gabriel M. Leung
}

The University of Hong Kong, Hong Kong, Hong Kong

\section{Objective}

This study evaluated the performance of an electronic smart-card based school absenteeism system in Hong Kong, 2008-2014.

\section{Introduction}

An electronic smart-card based school absenteeism surveillance system was introduced to Hong Kong since 2008. The pilot surveillance system initially began with 18 schools in 2008, and expanded to 107 schools in the current academic year of 2013-14. Data on all-cause absenteeism were collected from all participating schools and absenteeism due to sickness such as influenza-like illness, gastroenteritis and hand-foot-and-mouth disease were collected from $39(36.4 \%)$ schools.

Data collected were aggregated for the whole territory on a weekly basis for analysis. Temporal trend of influenza activity was disseminated with simple public health advice to each participating schools and the general public through a web-based dashboard [1]. These steps of data aggregation, analysis, and feedback report generation were automated by scripts in the software $\mathrm{R}$ which enhanced the timeliness and minimized workload required for maintaining the system.

\section{Methods}

We evaluated the school absenteeism system in the period from 1 January 2011 to 31 December 2013 after the system has become more stable. We examined its performance on several aspects, such as acceptability, simplicity, stability, resources requirement and data quality in terms of completeness, representativeness, timeliness and performance in reflecting elevated disease activity. A questionnaire survey was conducted in January 2014 in the participating schools which have joined the surveillance system and contributed for at least 3 years of data. 4 weeks of data were also randomly selected for assessment of data completeness.

\section{Results}

47 of the $72(65 \%)$ eligible participating schools responded to our survey. $80 \%$ of the responded schools considered that the system was easy to operate and can be easily learnt by other colleagues when needed, while $63.8 \%$ opined that it should be more widely used in local schools. While $44.7 \%$ of the schools reported their overall workload of staffs remained unchanged, $36.2 \%$ and $19.1 \%$ reported increased and decreased workload respectively by the use of this surveillance system.

The initial capital cost to install the eClass electronic school management system varied from as low as $\mathrm{HK} \$ 0-1,000$ (USD $0-128$ ) to $>\$ 50,000$ (USD 6,410). This had been considered as "very/ relatively expensive" or "very/relatively inexpensive" by the same proportion of responded schools (38.3\%). The main maintenance cost in the School of Public Health was manpower which cost for about $\$ 79,000$ (USD 10,128) annually.

Data reporting from four randomly selected weeks in 2013 (week 5, 10, 23 and 40) were examined which involved a total of 1602 data reporting files from 107 schools. The reporting of two data fields ("Number of all-cause absentees" and "Total number of enrolled student") were $100 \%$ complete from all participating schools. However, the reporting of data field "Number of ILI-specific absentees" was only $36.4 \%$ from all participating schools.

The system took around 4-5 working days from the data capturing to information dissemination. The longest time lag was about 2-3 days from data submission to data analysis, mainly to wait for data submission from the majority of the schools. Disease trends reflected by both the all-cause and ILI specific absenteeism rate closely followed the general rise, peak, and fall of influenza epidemic as measured by the pre-existing system. 3 of the 5 epidemic peaks matched exactly, and the remaining 2 peaks showed the school-based surveillance occurring 1-3 weeks before the peak shown by other systems.

\section{Conclusions}

The result demonstrated the feasibility and potential benefit of employing electronic school absenteeism data as captured automatically by a smart card system as an alternative data stream for monitoring influenza activities, and flexibility in establishing surveillance for emerging diseases. The increasing popularity of usage of smart card technology in various community settings might also represent potentially timely and cost-effective opportunities for innovative surveillance systems.

\section{Keywords}

school absenteeism; surveillance; electronic; evaluation

\section{References}

[1] Cheng CK, Ip DK, Cowling BJ, Ho LM, Leung GM, Lau EH. Digital dashboard design using multiple data streams for diseasesurveillance with influenza surveillance as an example. J Med Internet Res. 2011 Oct 14;13(4):e85.

\section{*Eric H.Y. Lau}

E-mail: ehylau@hku.hk 